\title{
ANÁLISIS BIOARQUEOLÓGICO DE LOS RESTOS DE ARROYO EL SIASGO (SUPUESTO HOMO CAPUTINCLINATUS DE AMEGHINO 1910)
}

\author{
Paula Escosteguy*, Clara Scabuzzo² y María Isabel González ${ }^{3}$
}

${ }^{I}$ CONICET-Instituto de Arqueología. Facultad de Filosofía y Letras. Universidad de Buenos Aires (UBA). Ciudad Autónoma de Buenos Aires (CABA). Argentina

${ }^{2}$ CONICET-Facultad de Ciencias Naturales y Museo. Universidad Nacional de La Plata. La Plata. Argentina

${ }^{3}$ Instituto de Arqueología. Facultad de Filosofía y Letras(UBA) Universidad de Buenos Aires. CABA. Argentina

PALABRAS CLAVE restos humanos; análisis paleodietario; fechado radiocarbónico; depresión del río Salado

\begin{abstract}
RESUMEN Las investigaciones arqueológicas desarrolladas en la Depresión del río Salado durante las últimas tres décadas, permitieron profundizar en el conocimiento de los grupos prehispánicos que vivieron allí durante el Holoceno tardío. Como resultado de los trabajos de campo en la microrregión, se conocen tres sitios con restos humanos (La Guillerma Ñandú, La Guillerma 1 y La Guillerma 5), totalizando un número mínimo de seis individuos inhumados de manera aislada. En esta contribución se suma, a la información precedente, el primer estudio bioarqueológico de un esqueleto recuperado por Carlos Ameghino en el arroyo El Siasgo a principios del siglo XX. El objetivo es presentar los resultados del análisis bioarqueológico de este esqueleto, así como el fechado radiocarbónico obtenido. Se busca así apor-
\end{abstract}

tar a la revisión de un caso de singular importancia histórica a través de la aplicación de un marco teórico y metodológico renovado. Como resultado de los análisis bioarqueológicos se pudo establecer que los restos corresponden a un individuo juvenil, de entre 12 y 15 años, que habría sido inhumado en posición primaria y que presentaba el cráneo deformado de manera circular. Se obtuvo, para este esqueleto, un fechado de $c a .3500$ años AP, que es el más antiguo para la depresión del río Salado. Los análisis isotópicos reflejan que la dieta del individuo fue mixta e incluyó la ingesta, en pequeñas cantidades, de alimentos marinos. Finalmente, estos resultados son integrados a la información bioarqueológica disponible para el área.Rev Arg Antrop Biol 19(2), 2017. doi:10.17139/raab.2017.0019.02.04

KEY WORDS human remains; paleodietary analysis; radiocarbon date; río Salado Depression

ABSTRACT The archaeological research developed in the Salado River Depression during the last three decades has offered knowledge about pre-Hispanic people who lived there along over the Late Holocene. As a result of fieldworks, human remains had been recovered from three archaeological sites (La Guillerma Ñandú, La Guillerma 1, and La Guillerma 5), resulting in a minimal number of six individuals, who had been buried separately. In this paper new information is presented, the first bioarchaeological study of the skeleton recovered by Carlos Ameghino in arroyo El Siasgo at the beginnings of the XX century. The aim of this work is to present the results of the bioarchaeological analysis of this skeleton, and the radiocarbon dating obtained. It thus, seeks

La antropología biológica así como la arqueología tuvieron, en la región pampeana, un inicio temprano a fines del siglo XIX, aunque su desenvolvimiento no fue constante ni homogéneo a lo largo del siglo XX (Politis, 1988). Los estudios del registro biológico humano desarrollados durante las primeras décadas del siglo XX contribuyeron, de manera secundaria, a la construcción del conocimiento sobre las antiguas poblaciones. Recién a fines de ese siglo, la bioarqueología, como una nueva línea de investigación, comenzó a jugar un papel importante en la comprensión de los estilos de vida de los grupos prehistóricos de la región. to the review of a singular historical case in the light of a renewed theoretical and methodological framework. From the bioarchaeological analysis, it was known that the human remains belonged to a juvenile (between 12 and 15 years old); that he appeared to have been buried in primary inhumation, and that his skull showed artificial cranial deformation. The dating obtained for the remains was $c a .3500$ years BP, which constituted the oldest dating for the Salado River Depression. The isotopic analysis showed a mixed diet that included the consumption -in low amounts- of sea food. Finally, the results of this research are integrated with other bioarchaeological data from the area. Rev Arg Antrop Biol 19(2), 2017. doi:10.17139/raab.2017.0019.02.04

La bioarqueología es un campo de conocimiento relativamente reciente, propuesto en

\footnotetext{
* Correspondencia a: Paula D. Escosteguy. CONICET-Instituto de Arqueología. Facultad de Filosofía y Letras. UBA. 25 de Mayo 217 3er piso. 1002 CABA. Argentina. E-mail: paueguy@hotmail.com

Financiamiento: Agencia Nacional de Promoción Científica y Tecnológica Proyecto PICT 2013-0411. Secretaría de Ciencia y Técnica de la Universidad de Buenos Aires Proyecto UBACyT 2014-2017 20020130100134BA.
}

Recibido 15 Febrero 2016; aceptado 21 Diciembre 2016

doi:10.17139/raab.2017.0019.02.04 
la década de 1970, con el objetivo de estudiar los restos humanos en relación con el contexto arqueológico (Larsen, 2002; Wright y Yoder, 2003; Agarwal y Glencross, 2011). Desde esta perspectiva, los restos humanos dejan de ser un anexo que explicaba una historia paralela a la arqueológica. Así, las investigaciones bioarqueológicas comienzan a ser integradas al momento de generar explicaciones arqueológicas (Agarwal y Glencross, 2011). En los últimos 20 años, el hallazgo y la excavación de sitios con entierros humanos permitieron el estudio de la biología de las poblaciones prehispánicas, a partir de la obtención de información con buena resolución contextual. El esqueleto humano proporciona la evidencia más directa de los aspectos físicos de las poblaciones y su estudio permite abordar tópicos tales como salud, violencia, dieta, demografía, estilos de vida y organización del trabajo, entre otros (Işcan y Kennedy, 1989; Molleson, 1994; Larsen, 2002).

Las investigaciones arqueológicas desarrolladas en la Depresión del río Salado durante las últimas tres décadas, dieron cuenta de los modos de vida de las poblaciones prehispánicas que allí vivieron durante el Holoceno tardío. Fueron grupos de cazadores-recolectores-pescadores que explotaron este humedal, asentaron sus campamentos en puntos altos del paisaje próximos al río, a los arroyos y a lagunas asociadas con este sistema hídrico (González, 2005; Frère, 2015). Como resultado de los trabajos de campo en la microrregión (sensu Aschero, 1988) correspondiente al sector bajo de la Depresión del Salado, se conocen tres sitios con restos humanos ( $\mathrm{La}$ Guillerma Nandú, La Guillerma 1 y La Guillerma 5; Fig. 1), que totalizan un número mínimo de seis individuos, inhumados de manera aislada. Para estos sitios se cuenta con cuatro fechados radiocarbónicos realizados sobre esqueletos humanos, que ubican a las inhumaciones en el Holoceno tardío (González, 2005; Scabuzzo y González, 2007; Frère et al., 2016). En esta contribución se suma, a la información precedente, el primer estudio bioarqueológico del esqueleto recuperado por Carlos Ameghino en el arroyo El Siasgo a principios del siglo XX.

El arroyo El Siasgo, en el sector próximo a su desembocadura en el río Salado, se caracteriza por la conformación de una laguna temporaria de ciclo aleatorio que se encuentra rodeada en el oeste por lomadas que se elevan entre 4 y 6 metros sobre el llano circundante (Dangavs y Blasi, 2002). Desde el año 2013 se están realizando investigaciones sistemáticas en este espacio. Ellas incluyeron prospecciones en ambas márgenes del arroyo (tanto recolección de superficie como revisión de las barrancas) y la realización de sondeos. Como producto de estos trabajos, se recuperaron tiestos de alfarería (fragmentos de cuerpo, borde y masas de arcillas cocidas), ítems líticos tales como instrumentos, núcleos, ecofactos y desechos -principalmente elaborados en ortocuarcita del Grupo Sierras Bayas (OGSB), en ftanita y caliza silicificaday escasos restos faunísticos (Escosteguy et al., 2015a). Aunque por el momento no se cuenta con dataciones para estos hallazgos, ellos muestran similitudes con los sitios ya excavados en el sector bajo de la Depresión del Salado, cuyas cronologías corresponden al Holoceno tardío (para más detalle véase González, 2005; Escosteguy et al., 2015a; Frère et al., 2016).

El objetivo de este trabajo es dar a conocer los resultados del análisis biarqueológico del esqueleto de arroyo El Siasgo -que actualmente forma parte de la colección Ameghino del Museo de Ciencias Naturales Bernardino Rivadavia- así como presentar el fechado radiocarbónico obtenido sobre muestras extraídas de este individuo. Se busca así aportar a la revisión de un caso de singular importancia histórica a través de la aplicación de un marco teórico y metodológico renovado. Finalmente, los resultados se discuten en relación con la información bioarqueológica disponible para la Depresión del río Salado.

\section{La Depresión del río Salado: el registro bioarqueológico, el ambiente y los conjuntos arqueofaunísticos}

En lo que se ha denominado como unidad espacial de análisis arqueológico Depresión del Salado, Politis y Barros (2006) han propuesto hacer una distinción entre la zona baja oriental y las llanuras altas. La zona oriental incluye el río Salado y el sistema de lagunas que en épocas de inundación se conectan con el río y sus afluentes, presenta un bosque continuo que está dominado por el tala (Celtis tala) y las especies que lo acompañan, que constituye recursos potenciales importantes. Por otro lado, hay una parte más 


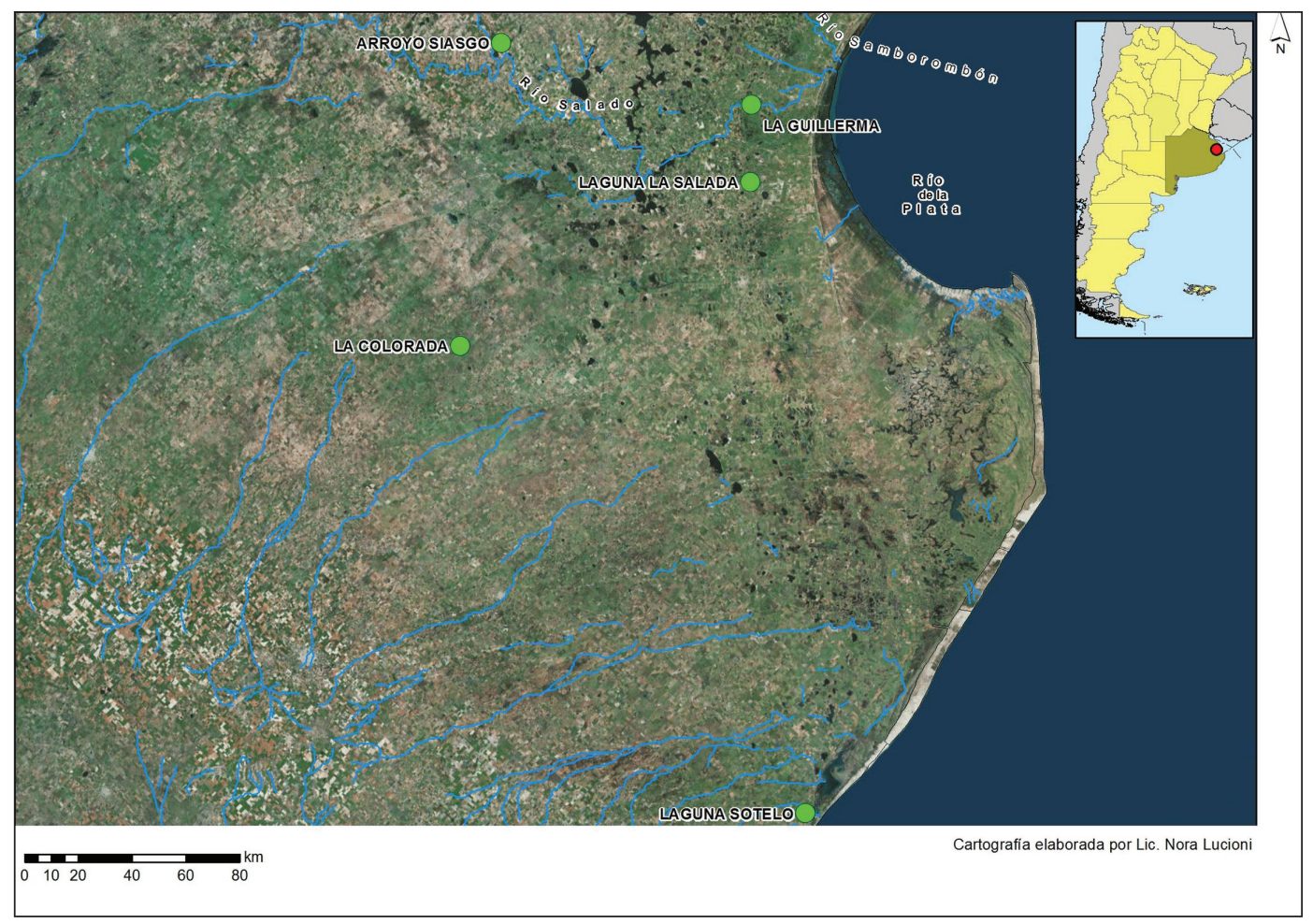

Fig. 1. Depresión del río Salado Bonaerense, sitios mencionados y arroyo El Siasgo.

alta que se extiende hasta las llanuras altas periféricas del Sistema Serrano de Tandilia y hacia las cotas más elevadas que limitan con la Pampa Ondulada. En ambas zonas de la Depresión del Salado se han detectado pocos sitios con restos humanos, por lo que el conocimiento bioarqueológico del área es escaso. Hasta el momento se conocen 6 sitios con registro bioarqueológico y un número mínimo de 17 individuos inhumados en el área (Tabla 1). Tres de los sitios (La Colorada, Laguna Sotelo y Laguna la Salada) se encuentran ubicados en la parte alta de la Depresión del Salado. Por su parte, los sitios de la localidad arqueológica La Guillerma están en la parte baja oriental con sus arroyos y lagunas. En cinco de los sitios de ambas zonas se obtuvieron fechados radiocarbónicos sobre restos humanos que indican episodios de inhumación a fines del Holoceno medio y en distintos momentos del Holoceno tardío, entre 3100 y 370 años AP.

El ambiente de humedal donde se recuperó el esqueleto aquí analizado se caracteriza por la presencia del río Salado, que es un curso de llanura con numerosos meandros y que incluye, en su cuenca, una gran cantidad de lagunas permanentes y temporarias con salinidad variable $(\mathrm{Ca}-$ nevari et al., 1998; Brinson y Malvárez, 2002). Gran parte de este ecosistema se encuentra temporal o permanentemente anegado a lo largo del año, lo cual produce una alta diversidad y productividad biótica (Bó y Malvárez, 1999).

La vegetación dominante es la estepa de gramíneas, existiendo también praderas, estepas samófilas y halófilas, bosques marginales y diversos tipos de vegetación hidrófila (como juncales de Schoenoplectus californicus y pajonales de Spartina densiflora). En la llanura de inundación se destaca la presencia de Schoenoplectus californicus (juncales), Zizaniopsis bonariensis (espadañales) y Typhas (totorales) mientras que, en los pantanos salinos, se registra Spartina brasiliensis (espartillares). En terrenos altos se encuentran los bosques de Celtis tala (tala), los cuales aquí se presentan en forma de islas y están asociados con otras especies arbóreas como Scutia buxifolia (coronillo), Jodina rhombifolia (sombra de toro), Cestrum parquii (duraznillo negro), Schinus longifolius (molle), 
TABLA 1. Información bioarqueológica de los sitios de la Depresión del río Salado

\begin{tabular}{|c|c|c|c|c|c|c|c|c|}
\hline Sitio & Edad ${ }^{14} \mathrm{C}$ AP & Código & $\begin{array}{l}\text { Grupo } \\
\text { etario }\end{array}$ & Sexo & NMI & $\begin{array}{c}\text { Deformación } \\
\text { del cráneo }\end{array}$ & $\begin{array}{l}\text { Modalidad de } \\
\text { inhumación }\end{array}$ & Fuente \\
\hline $\begin{array}{l}\text { Arroyo } \\
\text { El Siasgo }\end{array}$ & $3.590 \pm 25$ & $\begin{array}{c}\text { UGAMS } \\
22952\end{array}$ & SA & indet. & 1 & circular & primario? & este trabajo \\
\hline $\begin{array}{l}\text { La Guillerma } \\
\text { Ñandú }\end{array}$ & $1.640 \pm 40$ & CAMS-22030 & $\mathrm{A}$ & - & 1 & & $\begin{array}{l}\text { sin modalidad, } \\
\text { fragmentos } \\
\text { aislados }\end{array}$ & $\begin{array}{l}\text { González, } \\
2005\end{array}$ \\
\hline La Guillerma 1 & $410 \pm 40$ & CCNR-106303 & $A+S A$ & $\mathrm{M}+$ indet. & 3 & & $\begin{array}{c}\text { sin modalidad, } \\
\text { fragmentos } \\
\text { aislados }\end{array}$ & $\begin{array}{l}\text { González, } \\
2005\end{array}$ \\
\hline La Guillerma 5 & $370 \pm 40$ & Beta-13774 & A & - & 2 & & $\begin{array}{l}\text { sin modalidad, } \\
\text { fragmentos } \\
\text { aislados }\end{array}$ & $\begin{array}{l}\text { González, } \\
2005\end{array}$ \\
\hline La Colorada & $3.140 \pm 60$ & LP-807 & - & $\mathrm{F}+$ indet. & 3 & tabular oblicua & indet. & $\begin{array}{c}\text { Murgo y } \\
\text { Aldazabal, } \\
2007\end{array}$ \\
\hline $\begin{array}{l}\text { Laguna } \\
\text { La Salada }\end{array}$ & $1.470 \pm 50$ & LP-312 & $\mathrm{A}+\mathrm{SA}$ & M, F+ indet. & 7 & & primario & $\begin{array}{c}\text { Murgo y } \\
\text { Aldazabal, } \\
2007\end{array}$ \\
\hline $\begin{array}{l}\text { Laguna } \\
\text { Sotelo }\end{array}$ & sin datos & - & - & - & $1 ?$ & - & - & $\begin{array}{c}\text { Murgo y } \\
\text { Aldazabal, } \\
2007\end{array}$ \\
\hline
\end{tabular}

Referencias: $\mathrm{A}=$ =adulto; $\mathrm{SA}=$ subadulto; $\mathrm{M}=$ masculino; $\mathrm{F}=$ femenino; Indet.=indeterminado.

Sambucus australis (sauco) y Colletia spinosissima (brusquilla) (Gómez y Toresani, 1998; González, 2005; González y Frère, 2009).

En cuanto a la fauna, dentro de la clasificación zoogeográfica realizada por Ringuelet (1955), la Pampa bonaerense corresponde a los Dominios Pampásico y Subtropical. Los mamíferos característicos son Ozotoceros bezoarticus (venado de las pampas), varias especies de roedores, como Hydrochaeris hydrochaeris (carpincho), Myocastor coypus (coipo), Ctenomys $s p$. (tucu-tucu), armadillos y algunos carnívoros como el zorro gris (Lycalopex gimnocercus) (Burkart et al., 1999). En cambio, el ciervo de los pantanos (Blastocerus dichotomus) es una especie de la zona ribereña del Paraná-Plata y de origen subtropical que penetra ocasionalmente en la provincia de Buenos Aires (Cabrera, 1960).

Dentro de la ictiofauna se conocen 46 especies, siendo los Characiformes y Siluriformes los grupos dominantes. Esta cuenca constituye el límite meridional de la mayoría de las espe- cies de la ictiofauna Paraná-platense (López et al., 2001). Las aves son muy numerosas, con 274 especies identificadas en la actualidad. Dentro de esta clase, habitan el humedal tanto aves terrestres como acuáticas (Canevari et al., 1998), algunas de las cuales son migratorias. El ñandú (Rhea americana) se destaca como ave terrestre de gran tamaño, mientras que especies acuáticas muy abundantes son las garzas (familia Ardeidae), gallaretas (como Fulica leucoptera, Fulica armillata), biguás (Phalacrocorax olivaceus) y patos (familia Anatidae), entre otras (Canevari et al., 1998; Burkart et al., 1999).

Es relevante señalar que los materiales arqueofaunísticos recuperados en los sitios estudiados, también dan cuenta de la gran diversidad de especies presentes durante el Holoceno tardío. Algunas de ellas fueron explotadas por los grupos cazadores-recolectores-pescadores como coipo (M. coypus), venado de las pampas (O. bezoarticus), ciervo de los pantanos (B. dichotomus), zorro gris (L. gymnocercus), diversidad de aves (Familias Anatidae, Rallidae, Ardei- 
dae, etcétera) y numerosos peces (bagre -Rhamdia sapo-, tararira -Hoplias malabaricus-, entre otros) (González, 2005; Escosteguy et al., 2012, 2015b). Dentro de esta última clase, recientemente se propuso el aprovechamiento ocasional de dos especies estuariales como corvina negra (Pogonias cromis) y lisa (Mugil sp.) (González et al., 2016).

\section{MATERIAL Y MÉTODOS}

\section{El esqueleto de arroyo El Siasgo: contexto de hallazgo}

El sector donde se recuperó el esqueleto -sin otros materiales arqueológicos asociados- se encuentra en la margen izquierda del río Salado, entre los partidos de San Miguel del Monte y General Paz (Fig. 1). El hallazgo se realizó en la entonces llamada Estancia La Georgina (actualmente partido de General Paz) en las proximidades de la estación Villanueva del Ferrocarril del Sur (Ameghino, 1910a). Los trabajos de campo formaron parte de las expediciones organizadas por el Museo Nacional de Buenos Aires, cuyo director fue Florentino Ameghino desde abril de 1902 hasta su muerte, en agosto de 1911 (Podgorny, 1997).

Florentino Ameghino analizó el esqueleto recuperado por su hermano y a partir de su morfología craneana y de algunos huesos de las extremidades inferiores, propuso que se trataba de una nueva especie: Homo caputinclinatus (Ameghino, 1910b). Poco tiempo después del hallazgo, en su ponencia en el Congreso Científico Internacional Americano de 1910, presentó a esta especie como representante de un estadio intermedio entre Homo pampaeus y Homo sapiens. Cuando publicó esta presentación, el autor mencionó que los restos recuperados correspondían a un "esqueleto incompleto que se compone de cráneo sin mandíbula ni rostro debajo del frontal, algunos huesos largos, muchas vértebras y costillas, el sacro, la cadera, y varias articulaciones de los pies entre otras el astrágalo casi completo" (Ameghino, 1910a: 2-3).

En su descripción de los restos, Ameghino puntualizó que el cráneo tenía la frente fuyente dirigida hacia abajo, mientras que el agujero occipital se encontraba casi en la parte posterior del cráneo, lo cual indicaba, según el autor, que en vida el individuo había presentado un notable grado de inclinación hacia abajo de su cabeza (Ameghino 1910a, b, 1934). Asimismo otras particularidades del esqueleto que le indicaban su pertenencia a una especie homínida distinta, con un andar inclinado, eran: el cráneo pequeño y dolicocéfalo, vértex en posición muy posterior (semejante al cráneo de Homo pampaeus), capacidad craneana no superior a los $1000 \mathrm{cc}$, zona de inserción muscular del occipital poco marcada, suturas muy simples, casi rectas, apófisis mastoideas muy pequeñas y frontal de forma alargada y estrecha (semejante a Diprothomo). Para el postcráneo mencionó menor profundidad de la faceta del astrágalo, la línea áspera del fémur poco marcada y sección transversal del fémur, a mitad de diáfisis, de forma circular (Ameghino, 1910a, 1934).

Otros académicos de la época tuvieron acceso a los restos y propusieron ideas dispares a las de Florentino Ameghino (para más detalles véase Orquera, 1971). Algunos de ellos, como Aldobrandino Mochi y Aleš Hrdlička, señalaron que era un esqueleto de humano moderno y que el cráneo presentaba deformación intencional, no obstante que Ameghino hubiera considerado su morfología como propia de una especie antecesora (Ameghino, 1934:683). Hrdlička (1912) además propuso que esta deformación se correspondía con el tipo Aymará. Respecto de la edad, Ameghino consideraba que era un individuo joven (entre 16 y 18 años), con una talla reducida de aproximadamente 1,40 m (Ameghino, 1910a, b), mientras que sus colegas paleoantropólogos propusieron que se trataba de un niño de aproximadamente 12 años (Ameghino, 1910a, 1934; Hrdlička, 1912).

Un aspecto relevante y que ya fuera puntualizado por Orquera (1971), es que la asignación de este hallazgo a una especie humana precursora por parte de Ameghino se debió, en gran parte, a una errónea interpretación de la inclinación cefálica por la pérdida total de la región facial. Como consecuencia, se le asignó una postura incorrecta al individuo (Orquera, 1971:279).

Hrdlička (1912), incluso, solicitó al geólogo Bailey Willis -que lo acompañaba en su investigación sobre la antigüedad del Hombre en Sudamérica-, que se trasladara a observar el lugar de donde se extrajo el esqueleto. Allí, el personal de la Estancia La Georgina le indicó el sitio donde se habían exhumado los restos, ofreciendo información adicional sobre las condiciones 
del hallazgo. En su informe geológico, Willis sostuvo que el esqueleto se había encontrado dispuesto de norte a sur, con el cráneo levemente desplazado respecto de su posición original, en la ladera sur de una de las lomas de la estancia "about $2 m$ below the highest level of the plain, but covered by not more than half a meter of brown earth in place" (Willis, 1912:271). Este último dato discrepa con lo sostenido por Ameghino (1910a, 1934), quien había afirmado que se halló a $1.8 \mathrm{~m}$ de la superficie del suelo, en un depósito de loess, lo que según el autor apoyaba su gran antigüedad.

\section{Los estudios bioarqueológicos recientes}

Los análisis bioarqueológicos estuvieron orientados a realizar un inventario de los elementos óseos presentes, a la determinación de sexo y edad probables y al estudio paleodietario a partir de isótopos estables. En la realización del inventario se consignó: elemento, lateralidad, parte presente, grado de completitud, presencia de marcas de raíz, de carbonato y de óxido de manganeso. Para la determinación sexo-etaria se consideraron los criterios bioarqueológicos estándar (Buikstra y Ubelaker, 1994). Tanto los análisis isotópicos como los radiocarbónicos fueron realizados en el Center for Applied Isotope Studies (University of Georgia), para los cuales se seleccionaron fragmentos de costi1la. Para los análisis isotópicos, se solicitaron los valores de $\delta^{13} \mathrm{C}$ de la fracción orgánica e inorgánica, de $\delta^{15} \mathrm{~N}$ del colágeno y la relación de $\mathrm{C} / \mathrm{N}$ del colágeno de la muestra como indicador de fiabilidad isotópica. Los resultados isotópicos fueron analizados teniendo en cuenta los valores disponibles para los distintos recursos de la microrregión y de áreas vecinas.

Como ya fue demostrado, la composición isotópica de carbono y de nitrógeno de los diferentes tejidos de los animales refleja la composición isotópica de los alimentos consumidos. Los alimentos presentan diferentes valores de los isótopos de $\mathrm{C}^{13} / \mathrm{C}^{12}$ y N ${ }^{15} / \mathrm{N}^{14}$ dependiendo de diversas situaciones (Tykot et al., 2009; Froehle et al., 2012; Barberena, 2014). En el caso del carbono, la vía fotosintética de las plantas es uno de los factores que más influye en sus valores isotópicos. El carbono de la atmósfera se incorpora a los vegetales a través del proceso de fotosíntesis, que puede darse por distintas vías y que se vinculan con las plantas de tipo: $\mathrm{C}_{3}$, $\mathrm{C}_{4}$ o CAM. Según la vía utilizada hay un mayor o menor sesgo del isótopo más pesado, dando como resultado diferentes valores de la relación $\mathrm{C}^{13} / \mathrm{C}^{12}$. Los vegetales $\mathrm{C}_{3}$ (vía fotosintética Calvin-Benson) incluyen a todos los árboles y a arbustos y pastizales de ambientes templados y sus valores de $\delta^{13} \mathrm{C}$ son los más empobrecidos en $\mathrm{C}^{13}$. Por otro lado, los vegetales $\mathrm{C}_{4}$ (vía fotosintética Hatch-Slack) presentan los valores de $\delta^{13} \mathrm{C}$ más enriquecidos en $\mathrm{C}^{13} \mathrm{y}$ agrupan plantas adaptadas a zonas de altas temperaturas y humedad, como el maíz. Finalmente, las plantas CAM agrupan a los vegetales suculentos de ambientes áridos, con valores de $\delta^{13} \mathrm{C}$ intermedios (Pate, 1994). Estos valores isotópicos de los vegetales se transfieren a sus consumidores, aunque con diferencias debido al efecto del fraccionamiento isotópico. Como consecuencia, los grupos humanos presentan señales isotópicas diferentes de acuerdo con el tipo de vegetales y/o animales que formen parte de su alimentación.

Otro factor que provoca variaciones en los valores del carbono es el origen de los alimentos (terrestre o marino). En líneas generales, las cadenas tróficas marinas son más enriquecidas en $\delta^{13} \mathrm{C}$ que las terrestres; esto permite diferenciar el consumo de esos tipos de recursos a través de los análisis de isótopos estables de $\mathrm{C}$, si se trata de ambientes donde no hay vegetales del tipo $\mathrm{C}_{4}$ con valores que se superpongan a los de los recursos marinos (Katzenberg, 2008; Barberena, 2014).

Los análisis de $\delta^{13} \mathrm{C}$ se pueden realizar tanto en la fracción orgánica como en la inorgánica del hueso, obteniéndose en cada caso información diferente. Si bien ambas fracciones son apropiadas para evaluar la dieta total, los datos experimentales mostraron que los estudios realizados sobre el colágeno reflejan mejor el aporte proteico, en tanto la fracción inorgánica se correlaciona de forma más directa con la dieta total del individuo (i.e., proteína, carbohidratos y lípidos) (Ambrose y Kribaum, 2003; Froehle et al., 2012).

En relación con el nitrógeno, los análisis de $\delta^{15} \mathrm{~N}$ se usan para ver la ubicación de los individuos dentro de las cadenas tróficas, debido a que se da un aumento de los valores (o fraccionamiento) de 2 a $3 \%$ en cada nivel trófico (Froehle et al., 2012; Barberena, 2014). Se distinguen dos tipos de plantas según la manera en 
la que se da la absorción del nitrógeno. Por un lado, están las plantas fijadoras (mayormente leguminosas), que a partir de microorganismos radiculares transforman el nitrógeno gaseoso en aprovechable. En cambio, las plantas no fijadoras lo absorben del suelo en la forma de nitratos y amonios; este tipo de plantas poseen valores de $\delta^{15} \mathrm{~N}$ más enriquecidos en $\mathrm{N}^{15}$, con un rango entre 0 y 6\%, en comparación con las fijadoras (Pate, 1994; Killian Galván et al., 2015). Además, se debe tener en cuenta que otros factores como la aridez de los suelos y el uso de abonos orgánicos generan un aumento en estos valores isotópicos (Killian Galván et al., 2015).

Por otro lado, los alimentos marinos están más enriquecidos en $\mathrm{N}^{15}$ que los terrestres, (Barberena, 2014). Como una consecuencia lógica de esto, los grupos humanos cuyas dietas están sustentadas en el consumo de alimentos marinos presentan señales isotópicas de nitrógeno más enriquecidas que aquellos cuya alimentación es de origen terrestre. Finalmente, debido a que el hueso sufre varios procesos que pueden contaminarlo, se han desarrollado distintas estrategias de análisis que permiten conocer el grado de confiabilidad de los resultados isotópicos obtenidos. Entre los estudios más utilizados se encuentra el cálculo de la relación de $\mathrm{C} / \mathrm{N}$ en el hueso (Bocherens, 2000).

\section{RESULTADOS}

Como resultado del análisis bioarqueológico del individuo de arroyo El Siasgo se pudo establecer -como había indicado Ameghino (1910a)- que el esqueleto está incompleto: se encuentra representado por el cráneo, algunos huesos largos del miembro inferior, huesos del pie, ambos coxales fragmentados, el cúbito izquierdo, huesos de las manos, algunas vértebras y costillas (Fig. 2a). Con respecto a la edad de muerte se pudo determinar, a partir de la fusión de las epífisis, que se trataba de un individuo juvenil de entre 12 y 15 años de edad (sensu Buikstra y Ubelaker, 1994). No obstante, debido a la falta de partes diagnósticas, el sexo no pudo ser determinado. Este análisis también permitió determinar que el cráneo está deformado de manera circular, notándose una importante compresión mecánica sobre el frontal y el occipital (Fig. 2b). El resultado del fechado radiocarbó- nico realizado sobre una costilla ubica la inhumación en 3590 \pm 25 años AP (UGAMS 22952).

En cuanto a los resultados de los análisis isotópicos, el valor de la relación $\mathrm{C} / \mathrm{N}$ fue de 3.54 , que señala parámetros normales para la muestra. En cuanto a los resultados isotópicos, se obtuvo para el $\delta^{13} \mathrm{C}_{\text {col }}$ un valor de $-16.6 \%$, para $\delta^{13} \mathrm{C}_{\text {ap }}=-9.9 \%$ y para el $\delta^{15} \mathrm{~N}=13.3 \%$.
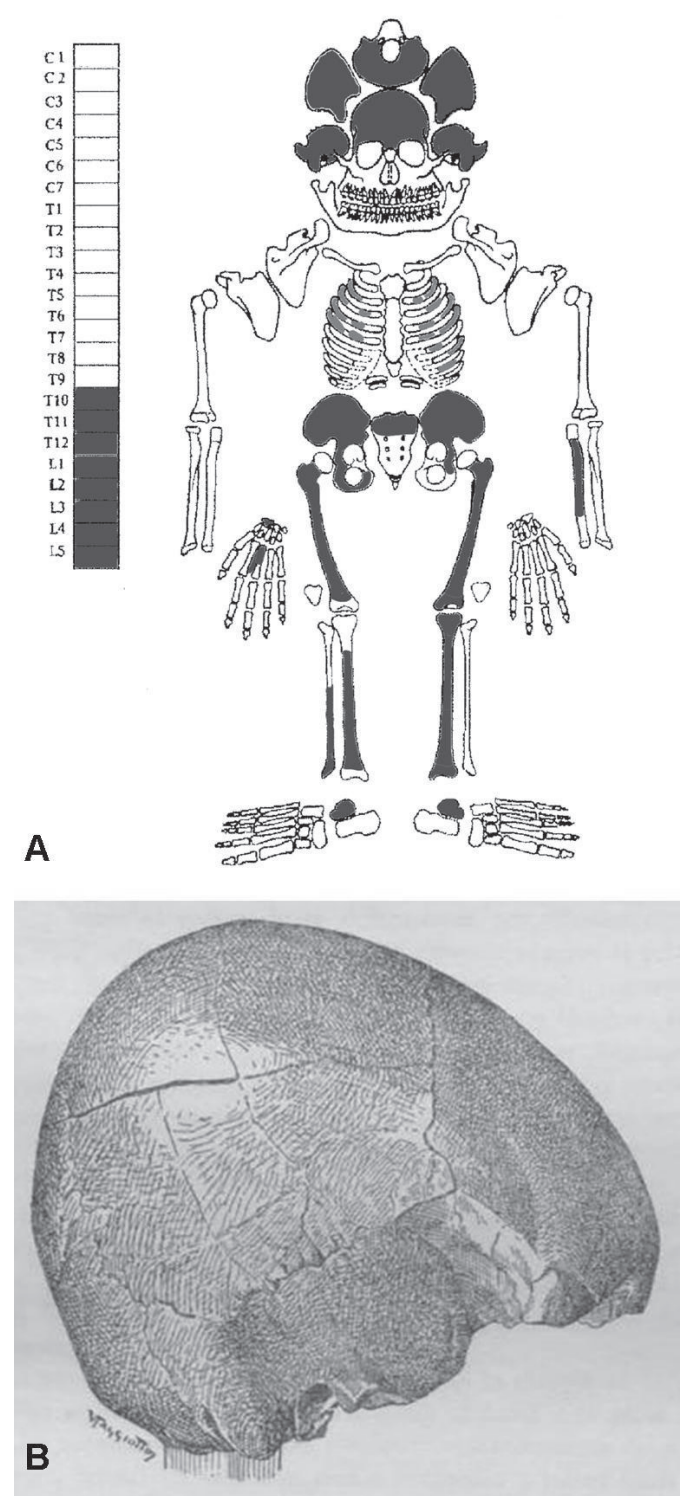

Fig. 2a. Esquema de las partes presentes en el esqueleto de arroyo El Siasgo. 2b. Dibujo del cráneo del individuo de arroyo El Siasgo. Tomado de Ameghino 1934, Lámina XI. 


\section{DISCUSIÓN}

El análisis aquí presentado se deriva de una colección conformada, con diferentes objetivos, hace más de cien años por parte de los hermanos Ameghino. No es la primera vez que en nuestro equipo de investigación se obtiene información sobre los antiguos pobladores de la Depresión del río Salado a partir de colecciones privadas o depositadas en museos locales. Por lo contrario, los materiales depositados en el Museo Pampeano de Chascomús, fueron el punto de partida para dar visibilidad pública al material de las poblaciones prehispánicas locales e impulsar el estudio arqueológico sistemático en la zona (para más detalle véase González, 2005; Salerno y Vigna, 2012; Salerno, 2014, entre otros). En este sentido, destacamos el valor cognitivo que poseen las colecciones de los museos y aún más cuando se pueden aplicar técnicas de análisis modernas. Así, al reconocer que el estudio de colecciones tiene ciertos alcances y limitaciones, y considerarlas como fuente de evidencia útil, se extraen datos significativos para la reconstrucción del pasado prehispánico (Bonomo et al., 2009). Además consideramos que un abordaje holístico que considera tanto la materialidad como la historia de la creación de la colección y el conocimiento generado por quienes la conformaron ofrece un valor agregado. En este caso, aunque Florentino Ameghino propuso de forma errónea al individuo del Siasgo como antecesor del hombre moderno, su análisis le permitió teorizar sobre el poblamiento pampeano. De esta manera, uno de los puntos importantes en el reanálisis de este individuo, es el fechado obtenido y presentado en este trabajo que amplía los rangos cronológicos para la cuenca del río Salado y representa la inhumación más antigua conocida hasta el momento.

Con respecto a las modalidades de inhumación presentes en el área, se reconocieron entierros primarios extendidos sólo en el sitio Laguna La Salada (Aldazabal, 2004). Para los sitios de la localidad La Guillerma no se pudo determinar si se trataba de inhumaciones primarias o secundarias, debido a que el registro bioarqueológico se compone únicamente de fragmentos del cráneo (Scabuzzo y González, 2007). En el caso de los restos de arroyo El Siasgo, si bien no se menciona en las publicaciones de F. Ameghino a qué tipo de inhumación corresponde (primaria o secundaria), hay ciertas características que indicarían que se trata de un entierro primario. Una de ellas es la descripción que recogió Willis (1912) de personas que participaron del hallazgo, quienes mencionaron la orientación del esqueleto y comentaron que el cráneo se encontraba levemente desplazado de su ubicación anatómica. Esta información, junto con la representación del esqueleto y la ausencia de marcas de corte y ocre -que son características frecuentes aunque no exclusivas de los entierros secundarios-, permiten proponer en conjunto que se trató de una inhumación primaria.

Los análisis del cráneo revelaron que el individuo presentaba una deformación con una importante compresión del frontal y del occipital, pudiendo ser incluido en el tipo circular (sensu Imbelloni, 1924-25). Baffi y Berón (1992) y posteriormente Barrientos (1997), han propuesto una secuencia cronológica para los diferentes tipos de deformaciones craneanas presentes en sitios pampeanos. Esta propuesta se basó en los resultados radiocarbónicos obtenidos para distintos sitios, sobre todo los correspondientes al sudeste pampeano y a la Pampa Seca. Según estas propuestas la deformación craneana del tipo circular sería la más antigua, con dataciones anteriores a 2000-3000 años AP, seguida por el tipo tabular oblicuo, fechado entre 3000 y 2000 años AP (Madrid y Barrientos, 2000; Politis et al., 2014) y finalmente, el tipo tabular erecto, con cronologías correspondientes a la última parte del Holoceno tardío (Berón y Baffi, 2003). Nuevos análisis radiocarbónicos realizados sobre cráneos con distintos tipos de deformación procedentes de sitios del nordeste de Patagonia corroboraron esta asociación entre tipo de deformación y cronología para este sector (Bernal et al., 2008; Mariano, 2011).

Por otro lado, la presencia de deformación craneana en el individuo aquí analizado resulta relevante, debido a que es el primer entierro del humedal del río Salado en el que se registró este tipo de modificación circular. En el área un antecedente de práctica deformatoria fue registrado en uno de los individuos del sitio La Colorada, el cual presentaba una deformación del tipo tabular oblicua (Aldazabal, 2004). En un futuro, el relevamiento de nuevos entierros con 
deformación craneana permitirá discutir, a nivel regional, estas modificaciones y su posible uso como indicador de la identidad de los individuos y de los grupos (Campillo, 2001).

En cuanto a los valores isotópicos, los resultados del $\delta^{13} \mathrm{C}$ colágeno se encuentran dentro de lo esperable para dietas mixtas basadas tanto en la ingesta de vegetales $\mathrm{C}_{3} \mathrm{y} \mathrm{C}_{4} \mathrm{y}$ animales consumidores de estos vegetales y/o la incorporación de alimentos de origen marino. Por su parte, el valor de $\delta^{13} \mathrm{C}$ de la fracción inorgánica indica el consumo de vegetales con vía fotosintética del tipo $\mathrm{C}_{4}$. Por último, el valor de $\delta^{15} \mathrm{~N}$ es coherente con la incorporación de proteínas de origen animal, tanto terrestres como marinas.

Finalmente, comparamos los resultados de los estudios paleodietarios aquí obtenidos con los datos disponibles para otros sitios con restos humanos de ambas zonas de la Depresión del Salado (Tabla 2). Cronológicamente, sólo el registro del sitio La Salada presenta un rango temporal similar al del esqueleto de arroyo El Siasgo, pues los otros sitios son más tardíos. En conjunto, estos valores muestran para el $\delta^{13} \mathrm{C}_{\text {col }}$ un rango situado entre -19.5 y $-14.2 \%$, para $\delta^{13} \mathrm{C}_{\text {ap }}$ entre -11.8 y $-9.8 \%$ y para el $\delta^{15} \mathrm{~N}$ entre 9.2 y 12.3\%o (Murgo y Aldazabal, 2007; Scabuzzo y González, 2007). Para el caso de los sitios del área costera, los resultados del análisis isotópico fueron interpretados en términos de dietas basadas en la ingesta de plantas $\mathrm{C}_{3}$ así como de herbívoros terrestres consumidores de este tipo de plantas. Por otro lado, los valores más enriquecidos en $\delta^{13} \mathrm{C}$ y $\delta^{15} \mathrm{~N}$ obtenidos para el sitio Laguna La Salada, fueron considerados como dietas mixtas basadas en alimentos derivados de $\mathrm{C}_{3}$ y alimentos marinos (Murgo y Aldazabal, 2007). En la microrregión de la Depresión del río Salado, los estudios paleodietarios reflejaron diferencias temporales en la alimentación: mientras que los valores de La Guillerma Ñandú, más empobrecidos en $\delta^{13} \mathrm{C}$, indican el consumo de plantas $\mathrm{C}_{3} \mathrm{y}$ de herbívoros terrestres consumidores de estos vegetales, los datos de los esqueletos más tardíos, con valores más enriquecidos en $\delta^{13} \mathrm{C}$, serían coherentes con el consumo de herbívoros de pequeño porte (como coipos), con dietas basadas en plantas $\mathrm{C}_{4}$ (Scabuzzo y González, 2007).

La diferencia en los recursos consumidos en la Depresión del Salado también se observa en los contextos arqueofaunísticos de cada uno de los sectores. Los coipos fueron muy importantes en los sitios del sector bajo (más del $40 \%$ del NISP en los sitios de la localidad La Guillerma y $56.7 \%$ del sitio SR7) junto con el aprovechamiento de peces, aves y en menor medida, cér-

TABLA 2. Información isotópica disponible para los sitios de la Depresión del río Salado con restos humanos

\begin{tabular}{cccccc}
\hline Sitio & $\begin{array}{c}\delta^{13} \mathrm{C}_{\mathrm{COL}} \\
(\%)\end{array}$ & $\begin{array}{c}\delta^{13} \mathrm{C}_{\mathrm{AP}} \\
(\%)\end{array}$ & $\begin{array}{c}\delta^{15} \mathrm{~N} \\
(\%)\end{array}$ & Tipo de dieta & Referencias \\
\hline Arroyo El Siasgo & -16.6 & -9.9 & 13.3 & mixta & este trabajo \\
La Guillerma Ñandú & -18.4 & -11.8 & 12.3 & continental C & Scabuzzo y González, 2007 \\
La Guillerma 1 & -15.6 & -10.5 & 11.2 & continental C & Scabuzzo y González, 2007 \\
La Guillerma 5 & -14.2 & -11.2 & 11.8 & continental $C^{4}$ & Scabuzzo y González, 2007 \\
La Colorada & -19.5 & -- & 9.2 & continental C & Murgo y Aldazabal, 2007 \\
Laguna La Salada & -17.6 & -- & 12.1 & mixta & Murgo y Aldazabal, 2007 \\
Laguna de Sotelo & -19.0 & -- & 10.4 & continental C & Murgo y Aldazabal, 2007 \\
\hline
\end{tabular}


vidos (González, 2005; Escosteguy et al., 2012). Por el contrario, en los sitios del sector alto se evidenció una estrategia alternativa: para el sitio Divisadero Monte 6, se infiere el consumo de micro roedores y peces, con mayor énfasis en especies marinas, además de una diversidad de taxa que fueron complementarios (Aldazabal et al., 2007). Mientras que, en el sitio Laguna Sotelo, se pudieron aprovechar especies mayores como Lama guanicoe, Ozotoceros bezoarticus y un cetáceo del suborden Mysticeti, además de otros recursos como armadillos y huevos de Rheidae (Aldazabal, 2004).

Para efectuar inferencias más confiables en relación con la dieta de los individuos, se analizaron los valores isotópicos de los recursos más abundantes en los sitios arqueológicos estudiados. Para complementar esta información, y debido a la escasez de datos para la Depresión del río Salado, también se consideraron los valores isotópicos de arqueofaunas de sitios del Delta del Paraná con cronología semejante (Tabla 3). Es importante aclarar que sólo se tuvieron en cuenta aquellas muestras para las cuales se contaba con información de $\delta^{13} \mathrm{Ccol}$ y $\delta^{15} \mathrm{~N}$.

Se cuenta con datos isotópicos para seis muestras: tres de ellas provienen del sitio $\mathrm{La}$ Guillerma 5 y tres de sitios del área del Delta del Paraná (Garín y Guazunambí: Loponte y Acosta, 2007; Loponte, 2008). Para los her- bívoros de porte grande ( $O$. bezoarticus), los valores isotópicos de $\delta^{13} \mathrm{Ccol}=-18.8 \%$ y de $\delta^{15} \mathrm{~N}=5.2 \%$ entran dentro del rango propuesto para herbívoros consumidores de vegetales C3. En cuanto a otros recursos, como los mamíferos de porte menor, se cuenta con dos muestras de coipo (M. coypus) con valores de $\delta^{13} \mathrm{Ccol}=-17.9$ y $-15.9 \%$ y de $\delta^{15} \mathrm{~N}=4.3$ y 4.5 $\%$. Estos valores indican el hábito herbívoro de este animal, basado en el consumo de vegetales tipo $\mathrm{C}_{3} \mathrm{y}$-en menor proporción- $\mathrm{C}_{4}$. Es importante mencionar que, en el ambiente donde se produjo el hallazgo, se encuentran disponibles varias especies de plantas $\mathrm{C}_{4}$ como gramíneas y juncos, siendo estos últimos la base de la alimentación del coipo (González, 2005). En lo que respecta a las aves de ambientes acuáticos, el valor de $\delta^{13} \mathrm{C}=-19.1 \%$ es cercano al disponible para venado de las pampas y el $\delta^{15} \mathrm{~N}=7 \%$ es uno de los más enriquecidos en N15 del conjunto. Finalmente, se tiene información isotópica de dos tipos de peces de agua dulce (Pimelodidae y $R$. sapo), con valores de $\delta^{13} \mathrm{Ccol}=-18$ y $-21.7 \%$ y $\delta^{15} \mathrm{~N}=6.11$ y $7.9 \%$, respectivamente; contándose estos últimos junto con las aves acuáticas ya señaladas, entre los más enriquecidos en $\delta^{15} \mathrm{~N}$ de la fauna considerada (González, 2005; Scabuzzo y González, 2007; Loponte, 2008).

En la Figura 3 se graficaron los valores isotópicos de $\delta^{13} \mathrm{Ccol}$ y $\delta^{15} \mathrm{~N}$ de las muestras huma-

TABLA 3. Valores isotópicos de especies faunisticas de sitios de la Depresión del Salado y sitios del Delta del Paraná

\begin{tabular}{|c|c|c|c|c|c|}
\hline Sitio & Especie & $\begin{array}{c}\delta^{13} \mathrm{C}_{\mathrm{COL}} \\
(\%)\end{array}$ & $\begin{array}{l}\delta^{15} \mathrm{~N} \\
(\%)\end{array}$ & $\begin{array}{l}\text { Código de } \\
\text { laboratorio }\end{array}$ & Referencias \\
\hline Guazunambí & $\begin{array}{l}\text { Ozotoceros } \\
\text { bezoarticus }\end{array}$ & -18.8 & 5.2 & UGA-9908 & Loponte, 2008 \\
\hline La Guillerma 5 & Myocastor coypus & -15.9 & 4.5 & CCNR-102542 & $\begin{array}{c}\text { Scabuzzo y González, } \\
2007\end{array}$ \\
\hline Garin & Myocastor coypus & -17.9 & 4.3 & & $\begin{array}{c}\text { Loponte y Acosta, } \\
2007\end{array}$ \\
\hline Garín & Pimelodidae & -18.1 & 6.1 & UGA-9909 & Loponte, 2008 \\
\hline La Guillerma 5 & Rhamdia sapo & -21.7 & 7.9 & CNR-102543 & \\
\hline La Guillerma 5 & $\begin{array}{l}\text { Ave indet. } \\
\text { (Rallidae o } \\
\text { Anatidae) }\end{array}$ & -19.1 & 7 & CNR-102541 & $\begin{array}{c}\text { Scabuzzo y González, } \\
2007\end{array}$ \\
\hline
\end{tabular}




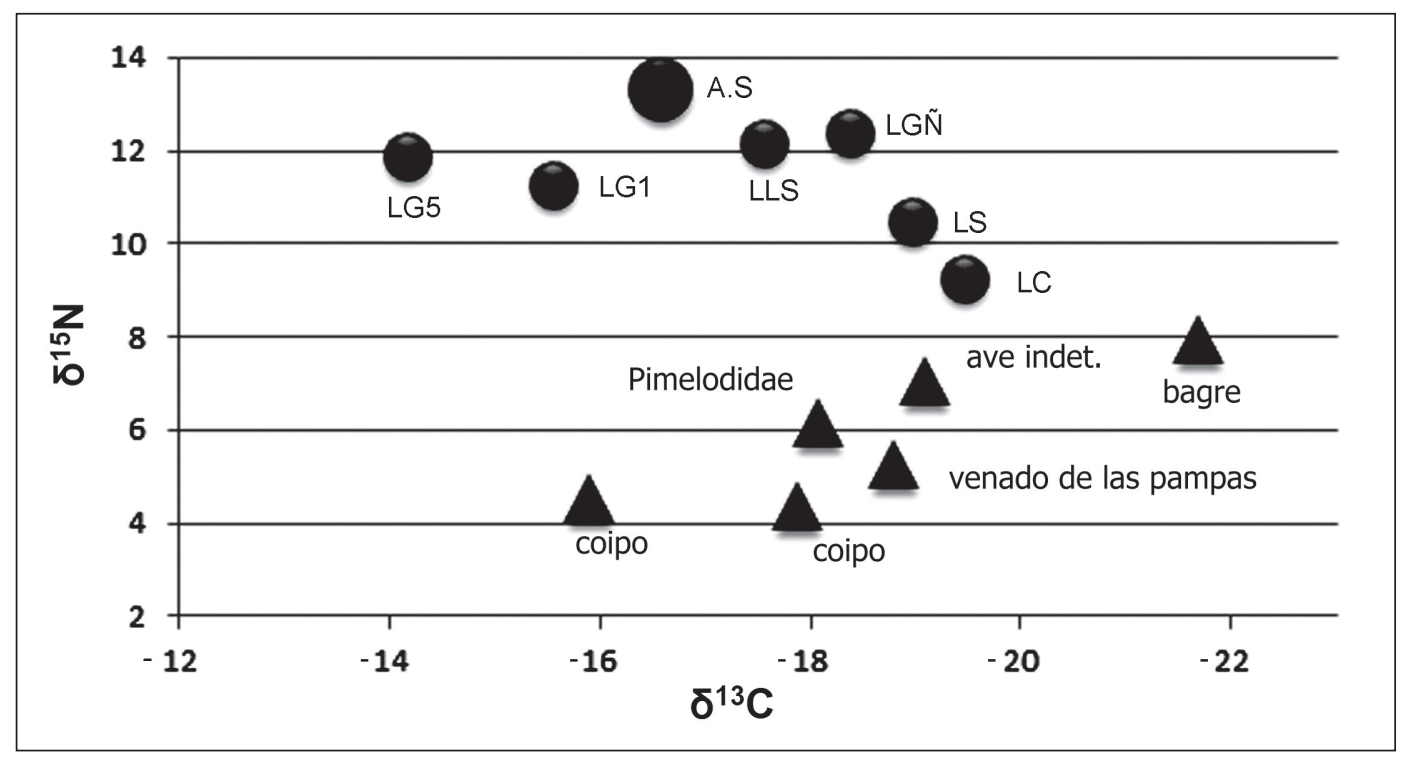

Fig. 3. Valores isotópicos de los esqueletos de la Depresión del Salado y de los recursos faunísticos más relevantes del área. Referencias: A.S:Arroyo El Siasgo; LGÑ:La Guillerma Ñandú; LG1:La Guillerma 1; LG5:La Guillerma 5; LLS:Laguna La Salada; LS:Laguna Sotelo; LC:La Colorada.

nas de los distintos sitios de la Depresión del Salado y de los recursos faunísticos. Teniendo en cuenta los valores de $\delta^{13} \mathrm{Ccol}$, se observa que la muestra humana de arroyo Siasgo tiene los valores más enriquecidos en $\mathrm{C}_{13}$ en comparación con la mayoría de los recursos, a excepción del coipo. Al tomar en cuenta el fraccionamiento isotópico que se da entre alimento y consumidor, es factible plantear que el valor de $\delta^{13} \mathrm{Ccol}$ del individuo se vincula con el consumo, no sólo de herbívoros terrestres consumidores de vegetales $\mathrm{C}_{3}$, sino también de aves de ambientes acuáticos continentales, coipo y peces. En relación con el $\delta^{13} \mathrm{Ccol}$, el valor del esqueleto de arroyo El Siasgo es cercano al promedio de valores disponibles para los otros esqueletos del área (-17.26\%) (Fig. 3). En cuanto a los datos de $\delta^{15} \mathrm{~N}$, el esqueleto aquí analizado presenta los valores más altos en comparación con las otras muestras humanas. En la Figura 3, entre los valores de la muestra humana y de los recursos (media $=5.8 \%$ ), se observa una distancia de $7.5 \%$, que es superior al fraccionamiento esperado entre consumidor y recurso. Esto podría estar indicando que se incorporó a la dieta, en forma significativa, algún recurso enriquecido en $\mathrm{N}_{15}$, como por ejemplo alimentos de origen marino.
Como ya se hizo mención, los resultados isotópicos obtenidos indican que la dieta del individuo fue mixta y se basó en herbívoros consumidores de vegetales $\mathrm{C}_{3}$, peces de agua dulce, aves y en la ingesta complementaria de alimento marino. El consumo de alimento marino es coherente con la distancia del sitio a la costa, aproximadamente $100 \mathrm{~km}$ en la actualidad. Sin embargo, es interesante notar que sólo se han registrado escasos restos arqueofaunísticos de animales marinos en los sitios de la localidad arqueológica La Guillerma (Pogonias cromis y Mugil sp.), aunque hay que considerar que estos son, en su mayoría, de momentos más tardíos y no se conocen hasta el momento contextos faunísticos con cronología como la de arroyo El Siasgo (González et al., 2016). En este sentido, no se podría descartar la captura de especies estuariales que ingresan al río Salado. Otra posibilidad es que estos grupos hayan tenido temporadas de residencia en la costa durante las cuales aprovecharan estos recursos marinos, tanto por pesca como en eventos ocasionales de mortandad masiva, tal como proponen Politis y Leon (2010). Por último, otra alternativa menos probable es que los valores isotópicos se deban al consumo de otros recursos no detectados hasta el momento. 


\section{CONCLUSIONES}

El estudio de este esqueleto adquiere relevancia en dos aspectos. En primer lugar, permite recuperar nueva información referida al estudio de las poblaciones prehispánicas de la Depresión del río Salado bonaerense. El segundo aspecto, se vincula con la escasez de esqueletos humanos en el sector de estudio. En este sentido, los restos humanos hallados en arroyo El Siasgo constituyen un aporte novedoso al tratarse del entierro más completo para la microrregión, con presencia de elementos óseos postcraneanos $\mathrm{y}$, en el cual, se ha podido registrar deformación intencional del cráneo. En cuanto a los aspectos cronológicos, el fechado obtenido para el que fuera denominado Homo caputinclinatus (sensu Ameghino, 1910b) es el más temprano para la microrregión, lo que sitúa a las evidencias más tempranas de ocupación humana del área en 3500 años AP. Finalmente, aunque nuestra interpretación difiere con la realizada por Florentino Ameghino, los resultados que aquí presentamos tienen un valor histórico, ya que se trata de restos humanos dados a conocer hace más de un siglo y que formaron parte de su propuesta sobre la existencia de antecesores humanos en las pampas argentinas (Ameghino, 1910a, b). En conclusión, nos interesa destacar que las opiniones vertidas en el pasado pueden y deben ser revisadas a la luz de nuevos enfoques, facilitados por los métodos de análisis disponibles actualmente.

\section{AGRADECIMIENTOS}

Al Dr. Mariano Bonomo (FCNyM, UNLPCONICET), quien nos orientó en la búsqueda del esqueleto. Al personal del MACN-CONICET (Dr. Kramarz y Stella Maris Álvarez) por permitir el acceso y análisis de los materiales. Al Dr. Mariano Del Papa (FCNyM, UNLP) por el análisis de la deformación craneana. Al Dr. Ricardo Bonini (INCUAPA-CONICET) por su aporte bibliográfico y su colaboración en el campo. La Dra. Virginia Salerno (IA, FFyL, UBA-CONICET) contribuyó con la búsqueda de documentación histórica, en trabajos de campo y realizó importantes sugerencias. Al Lic. Luis Orquera y a la Dra. Magdalena Frère por sus valiosos comentarios y aportes sobre versiones previas del manuscrito. A los evaluadores, por sus recomendaciones que contribuyeron a mejorar este artículo.

\section{LITERATURA CITADA}

Agarwal S, Glencross B, editores. 2011. Social Bioarchaeology. Oxford: Wiley-Blackwell. doi: 10.1002/9781444390537

Aldazabal V. 2004. La ocupación humana en el sector centro oriental de la Pampa Deprimida. Tesis Doctoral inédita. Facultad de Filosofia y Letras. Universidad de Buenos Aires.

Aldazabal V, Eugenio E, Silveira M. 2007. Zooarqueología del sitio "El Divisadero Monte 6". Partido de General Lavalle, provincia de Buenos Aires. En: Libro de resúmenes del XVI Congreso Nacional de Arqueología Argentina. Jujuy: EDIUnJu. Tomo III, p 241-246.

Ambrose S, Kribaum J. 2003. Bone chemistry and bioarchaeology. J Anthropol Archaeol 22(3):191-192. doi:10.1016/S0278-4165(03)00032-1

Ameghino F. 1910a. Descubrimiento de un esqueleto fósil en el Pampeano superior del arroyo Siasgo. Separata del Congreso Científico Internacional Americano. Buenos Aires: Imprenta y Casa Editora "Juan A. Alsina". p 1-6.

Ameghino F. 1910b. Geología, paleogeografía, paleontología, antropología. En: Diario La Nación, Edición especial por el Centenario de la Patria. 25 de Mayo de 1910 Buenos Aires. p 174-180.

Ameghino F. 1934 Descubrimiento de un esqueleto fósil en el Pampeano superior del arroyo Siasgo. En: Torcelli A, compilador. Paleoantropología argentina. Obras Completas y correspondencia científica de Florentino Ameghino. La Plata: Taller de Impresiones Oficiales. Volumen XVIII. p 409-413.

Aschero C. 1988. De punta a punta: producción, mantenimiento y diseño en puntas de proyectil precerámicas de la Puna argentina. Precirculados del IX Congreso Nacional de Arqueología Argentina. Simposio Las unidades de análisis para el estudio del cambio cultural en arqueología. p 219-229. Buenos Aires.

Baffi EI, Berón M. 1992. Los restos óseos humanos de Tapera Moreira (La Pampa), y la deformación artificial en la región Pampeana. Análisis tentativo. Palimpsesto 1: 25-36.

Barberena R. 2014. Isotopic studies of foragers' diet: environmental archaeological approaches. En: Smith C. editor. Encyclopedia of global archaeology. New York: Springer. p 4111-4120. doi:10.1007/978-1-4419-0465-2

Barrientos G. 1997. Nutrición y dieta de las poblaciones aborígenes prehispánicas del sudeste de la región Pampeana. Tesis doctoral inédita. Facultad de Ciencias Naturales y Museo. Universidad Nacional de La Plata.

Bernal V, González P, Pérez S, Pucciarelli H. 2008. Entierros humanos del noreste de patagonia: nuevos fechados radiocarbónicos. Magallania 36(2):175-183.

Berón M, Baffi EI. 2003. Procesos de cambio cultural en los cazadores recolectores de la provincia de La Pampa, Argentina. Intersecciones Antropol. 4:29-45.

Bó R, Malvárez AI. 1999. Las inundaciones y la Biodiversidad en humedales. Un análisis del efecto de eventos extremos sobre la Fauna silvestre. En: Malvárez AI, editor. Tópicos sobre humedales subtropicales y templados de Sudamérica. Montevideo: MAB-ORCYT. p 147-68. Bocherens R. 2000. Isotopic signals $\left(\mathrm{C}_{13} ; \mathrm{N}_{15}\right)$ in Pleistoce- 
ne mammals. En: Ambrose SH, Katzenberg MA, editores. Biogeochimical approches to paleodietary análisis. Nueva York/Londres: Plenum Publishers. p 65-84.

Bonomo M, Capdepont I, Matarrese A. 2009. Alcances en el estudio de colecciones. Los materiales arqueológicos del Delta del río Paraná depositados en el Museo de La Plata (Argentina). Arqueología Sudamericana 5:68-101.

Brinson M, Malvárez AI. 2002. Temperate freshwater wetlands: types, status, and threats. Environ Conserv J 29(2): 115-133. doi:10.1017/S0376892902000085

Buikstra JE, Ubelaker DH. 1994. Standars for data collection from human skeletal remains. Series $\mathrm{N}^{\circ} 44$. Arkansas: Ark. Archaeol. Sur. Res.

Burkart R, Bárbaro NO, Sánchez RO, Gómez DA. 1999. Eco-regiones de la Argentina. Buenos Aires: Administración de Parques Nacionales.

Cabrera AL. 1960. Catálogo de los mamíferos de América del Sur. Revista Museo Argentino de Ciencias Naturales Bernardino Rivadavia Zoología 4 (1):309-732.

Campillo D. 2001. Introducción a la Paleopatología. Barcelona: Bellaterra.

Canevari P, Blanco DE, Bucher EH, Castro G, Davidson I. 1998. Los humedales de la Argentina. En: Canevari P, Blanco DE, Bucher EH, Castro G, Davidson I, compiladores. Humedales de la Argentina. Clasificación, situación actual, conservación y legislación. Buenos Aires: Humedales Internacional y Secretaría de Recursos Naturales y Desarrollo Sustentable. p 1-24.

Dangavs N, Blasi A. 2002. Los depósitos de yeso intrasedimentario del arroyo El Siasgo, partidos de Monte y General Paz, provincia de Buenos Aires. Revista de la Asociación Geológica Argentina 57 (3):315-327.

Escosteguy P, Salemme M, González MI. 2012. Myocastor coypus ("coipo", Rodentia, Mammalia) como recurso en los humedales de la Pampa bonanerense: patrones de explotación. Revista del Museo de Antropología 5:13-30.

Escosteguy P, Salerno V, Granda P, Vigna M. 2015a. Primeros resultados de las investigaciones arqueológicas en Arroyo El Siasgo (Depresión del río Salado, Buenos Aires). Relaciones XL (2):645-653.

Escosteguy P, González MI, Frère MM. 2015b. Nuevos datos sobre fauna menor de la Depresión del Río Salado (Provincia de Buenos Aires, Argentina): el caso de San Ramón 7. Archaeofauna. International Journal of Archaeozoology 24:295-313.

Frère MM. 2015. Tecnología cerámica de los cazadoresrecolectores-pescadores de la microrregión del río Salado, provincia de Buenos Aires. Tesis Doctoral Inédita. Facultad de Filosofía y Letras. Universidad de Buenos Aires.

Frère MM, González MI, Greco C. 2016. Continuity in the use of shallow sites of the Salado river basin in the Pampean region, Argentina. Radiocarbon 58(4):921-933. doi: $10.1017 /$ RDC. 2016.63

Froehle AW, Kellner CM, Schoeninger MJ. 2012. Multivariate carbon and nitrogen stable isotope model for the reconstruction of prehistoric human diet. Am J Phys Anthropol. 147:352-369. doi:10.1002/ajpa.22088

Gómez SE, Toresani NI. 1998. Pampas. En: Canevari P, Blanco DE, Bucher EH, Castro G, Davidson I, compiladores. Humedales de la Argentina. Clasificación, situación actual, conservación y legislación. Buenos Aires: Humedales Internacional y Secretaría de Recursos Naturales y Desarrollo Sustentable. p 97-114.

González MI. 2005. Arqueología de alfareros, cazadores y pescadores pampeanos. Buenos Aires: Sociedad Argentina de Antropología.

González MI, Frère MM. 2009. Talares y paisaje fluvial bonaerense: arqueología del río Salado. Intersecciones Antropol 10:249-265.

González MI, Escosteguy P, Frère MM. 2016. Estudio ictioarqueológico y la presencia de corvina negra en La Guillerma 5 (Depresión del río Salado, provincia de Buenos Aires). Actas del III Encuentro Latinoamericano de Zooarqueología. Aracajú. Brasil.

Hrdlička A. 1912. Early man in South America. Número 52. Washington: Smithsonian Institution. Bureau of American Ethnology. p 269-272.

Imbelloni J. 1924-25. Deformaciones intencionales del cráneo en Sud América. Revista del Museo de La Plata 28:329-407.

Işcan M, Kennedy K. 1989. Reconstruction of life from the skeleton: an introduction. En: Işcan M, Kennedy K, editores. Reconstruction of life from the skeleton. Nueva York: Alan R. Liss, Inc. p 1-10.

Katzenberg A. 2008. Stable isotope analysis: a tool for studying past diet, demography, and life history. En: Katzenberg MA, Saunders S, editores. Biological anthropology of the human skeleton. New Jersey: Wiley-Liss. p 413-443.

Killian Galván V, Sanmartino A, Castellano V, Seldes V, Marban L. 2015. Estudios de isótopos estables en huertas actuales de la Quebrada de Humahuaca (Jujuy, Argentina). Su potencial aporte a los estudios paleodietarios del Noroeste Argentino. Revista del Museo de Antropología 8(2):107-118.

Larsen CS. 2002. Bioarchaeology: The lives and lifestyles past people. J Archaeol Res 10(2):119-153. doi:10.1023/A:1015267705803

López HL, Baigún CRM, Iwaszkiw JM, Delfino RL, Padin OH. 2001. La cuenca del Salado: uso y posibilidades de sus recursos pesqueros. La Plata: Editorial de la Universidad de La Plata.

Loponte D. 2008. La economía prehistórica del norte bonaerense. Arqueología del humedal del Paraná inferior, los Bajíos Ribereños meridionales. Tesis Doctoral Inédita. Facultad de Ciencias Naturales y Museo. Universidad Nacional de La Plata.

Loponte D, Acosta A. 2007. Horticultores amazónicos en el humedal del Paraná Inferior: los primeros datos isotópicos de la dieta. En: Bayón C, González MI, Flegenheimer N, Pupio A, Frére MM, editores. Arqueología en las Pampas: Buenos Aires: Sociedad Argentina de Antropología. p 75-94.

Madrid P, Barrientos G. 2000. La estructura del registro arqueológico del sitio Laguna Tres Reyes 1 (provincia de Buenos Aires): nuevos datos para la interpretación del poblamiento humano del sudeste de la región Pampeana a inicios del Holoceno tardío. Relaciones XV:179-206.

Mariano CI. 2011. Prácticas mortuorias y registro bioarqueológico en la costa rionegrina del golfo San Matías, Argentina. Intersecciones Antropol. 12:17-30.

Molleson T. 1994. The eloquent bones of Abu Hureya. Sci Am. 271(2):70-75. doi:10.1038/scientificamerican0894-70

Murgo A, Aldázabal V. 2007. Análisis de isótopos estables de carbono y nitrógeno de los restos óseos humanos provenientes de sitios área Pampa Deprimida centro oriental. En: Oliva F, de Grandis N, Rodríguez J, editores. Arqueología Argentina en los inicios de un nuevo siglo. Rosario: Universidad Nacional de Rosario. p 259-264.

Orquera LA. 1971. Paleoantropología de la Pampa húmeda. Tesis de Licenciatura Inédita. Facultad de Filosofía y Letras. Universidad de Buenos Aires.

Pate D. 1994. Bone chemistry and paleodiet. J Archaeol Method Th 1(2):161-209. doi:10.1007/BF02231415 
Podgorny I. 1997. De la santidad laica del científico Florentino Ameghino y el espectáculo de la ciencia en la Argentina moderna. Entrepasados 13:37-61.

Politis G. 1988. Paradigmas, modelos y métodos en la Arqueología de la Pampa Bonaerense. En: Yacobaccio H, compilador. Arqueología Argentina Contemporánea: Buenos Aires: Editorial Búsqueda. p 59-107.

Politis G, Barros P. 2006. La región pampeana como unidad espacial de análisis en la arqueología contemporánea. Folia Histórica del Nordeste 16: 51-73.

Politis G, Barrientos G, Scabuzzo C. 2014. Los entierros humanos de Arroyo Seco 2. En: Politis G, Gutiérrez MA, Scabuzzo C, editores. Estado Actual de la Investigaciones en el sitio arqueológico Arroyo Seco 2 (región pampeana, Argentina). Olavarría: Facultad de Ciencias Sociales, UNCPBA. Serie Monográfica INCUAPA 6. p 329-369.

Politis G, Leon DC. 2010. Patrones adaptativos de los cazadores-recolectores-pescadores de la margen occidental del Paraná inferior-Plata. En: Cocco G, Feuillet Terzaghi MR, editores. Arqueología de cazadores recolectores en la cuenca del Plata. Santa Fe: Centro de Estudios Hispanoamericanos. p 63-86.

Ringuelet R. 1955. Panorama zoogeográfico de la provincia de Buenos Aires. Notas del Museo de La Plata 18 (156):1-45.
Salerno V. 2014. Trabajo arqueológico y representaciones del pasado en la provincia de Buenos Aires. Buenos Aires: Editorial de la Facultad de Filosofía y Letras. Universidad de Buenos Aires.

Salerno V, Vigna M. 2012. Acercamiento a la construcción del pasado prehispánico en una sala del Museo Pampeano de Chascomús entre 1939 y 1992. Arqueología 18:181-207.

Scabuzzo C, González MI. 2007. Un acercamiento a la dieta de las poblaciones prehispánicas de la depresión del Salado durante el Holoceno tardío. En: Bayón C, Flegenheimer N, González MI, Pupio A, Frère MM, editores. Arqueología en las pampas: Buenos Aires: Sociedad Argentina de Antropología. p 59-73.

Tykot R, Falabella F, Planella M, Aspillaga E, Sanhueza L, Becker C. 2009. Stable isotopes and archaeology in Central Chile: methodological insights and interpretative problems for dietary reconstruction. Int J Osteoarch 19(2):156-170. doi:10.1002/oa.1065

Willis B. 1912. Observations on the Arroyo Siasgo find. En: Hrdlička A. Early man in South America. Número 52. Washington: Smithsonian Institution. Bureau of American Ethnology. p 269-272.

Wright L, Yoder C. 2003. Recent progress in bioarchaeology: approaches to the osteological paradox. J Archaeol Res 11(1):43-70. doi:10.1023/A:1021200925063 